\title{
Phonon-Pump Extreme-Ultraviolet-Photoemission Probe in Graphene: Anomalous Heating of Dirac Carriers by Lattice Deformation
}

\author{
Isabella Gierz, ${ }^{1, *}$ Matteo Mitrano, ${ }^{1}$ Hubertus Bromberger, ${ }^{1}$ Cephise Cacho, ${ }^{2}$ Richard Chapman, ${ }^{2}$ Emma Springate, ${ }^{2}$ \\ Stefan Link, ${ }^{3}$ Ulrich Starke, ${ }^{3}$ Burkhard Sachs, ${ }^{4}$ Martin Eckstein, ${ }^{1}$ Tim O. Wehling, ${ }^{5}$ Mikhail I. Katsnelson, ${ }^{6}$ \\ Alexander Lichtenstein, ${ }^{4}$ and Andrea Cavalleri ${ }^{1,7}$ \\ ${ }^{1}$ Max Planck Institute for the Structure and Dynamics of Matter, Center for Free Electron Laser Science, 22761 Hamburg, Germany \\ ${ }^{2}$ Central Laser Facility, STFC Rutherford Appleton Laboratory, OX11 OQX Harwell, United Kingdom \\ ${ }^{3}$ Max Planck Institute for Solid State Research, 70569 Stuttgart, Germany \\ ${ }^{4}$ I. Institut für Theoretische Physik, Universität Hamburg, 20355 Hamburg, Germany \\ ${ }^{5}$ Institut für Theoretische Physik, Universität Bremen, 28359 Bremen, Germany \\ ${ }^{6}$ Institute for Molecules and Materials, Radboud University Nijmegen, 6525 HP Nijmegen, Netherlands \\ ${ }^{7}$ Department of Physics, Clarendon Laboratory, University of Oxford, OX1 3PU Oxford, United Kingdom
}

(Received 14 November 2014; published 25 March 2015)

\begin{abstract}
We modulate the atomic structure of bilayer graphene by driving its lattice at resonance with the in-plane $E_{1 u}$ lattice vibration at $6.3 \mu \mathrm{m}$. Using time- and angle-resolved photoemission spectroscopy (tr-ARPES) with extreme-ultraviolet (XUV) pulses, we measure the response of the Dirac electrons near the $K$ point. We observe that lattice modulation causes anomalous carrier dynamics, with the Dirac electrons reaching lower peak temperatures and relaxing at faster rate compared to when the excitation is applied away from the phonon resonance or in monolayer samples. Frozen phonon calculations predict dramatic band structure changes when the $E_{1 u}$ vibration is driven, which we use to explain the anomalous dynamics observed in the experiment.
\end{abstract}

DOI: 10.1103/PhysRevLett.114.125503

PACS numbers: 63.22.Rc, 73.22.Pr, 79.60.-i

Optical excitation of Dirac carriers in graphene has to date been known to occur through two mechanisms. For photon energies higher than twice the chemical potential $\left(\hbar \omega_{\text {pump }}>2\left|\mu_{e}\right|\right)$, direct interband excitation takes place $[1,2]$, resulting in population inversion for sufficiently high fluences [3-5]. For doped samples and lower photon energies $\left(\hbar \omega_{\text {pump }}<2\left|\mu_{e}\right|\right)$, the Dirac carriers are heated by metallic free carrier absorption $[4,6]$, where the peak electronic temperature is determined by the pump fluence [4].

Here, we introduce a new mechanism, active when an infrared optical field is made resonant with a vibrational mode that modulates the band structure. We show that anomalous heating of the Dirac carrier distribution occurs when the $E_{1 u}$ phonon mode of bilayer graphene [7-13] is driven to large amplitudes with a coherent midinfrared (MIR) field. This mode is particularly interesting as it exhibits a pronounced Fano profile and large oscillator strength in the conductivity spectrum, originating from an anomalously strong coupling to electronic interband transitions [14-16]. Further, the $E_{1 u}$ motion of the two triangular lattice units [Fig. 1(a)] is expected to periodically open and close a band gap at the $K$ point [17], a prospect of general interest for the physics of graphene. Finally, the frequency of the mode is fast compared to the electronphonon scattering time, resulting in a breakdown of the adiabatic Born-Oppenheimer approximation as proposed in the context of Raman scattering experiments for monolayer graphene [18].
Quasifreestanding epitaxial graphene mono- and bilayers, grown on the silicon-terminated face of silicon carbide (SiC), are used in this work [19] (see Supplemental Material [20]). The equilibrium ARPES spectra for these samples, measured at room temperature with $\operatorname{HeII} \alpha$ radiation at $\hbar \omega=40.8 \mathrm{eV}$, are shown in Fig. 1(b). These measurements were performed along a momentum cut that crosses the $K$ point perpendicular to the $\Gamma K$ direction [inset Fig. 1(b)], and show the characteristic $\pi$-band dispersion of graphene. Both samples are lightly hole doped due to charge transfer from the substrate with hole concentrations of $n=6 \times 10^{12} \mathrm{~cm}^{-2}$ and $n=4 \times 10^{12} \mathrm{~cm}^{-2}$ in monolayer and bilayer graphene, respectively.

MIR pulses were used to excite these samples, either resonantly with the $E_{1 u}$ mode in bilayer graphene $\left(\lambda_{\text {pump }}=6.3 \mu \mathrm{m}\right)$ or at other wavelengths between 4 and $9 \mu \mathrm{m}$. The resulting nonequilibrium Dirac carrier distributions were tracked with tr-ARPES as a function of pump-probe time delay using XUV femtosecond pulses at $31 \mathrm{eV}$ photon energy. The experiments were performed at $30 \mathrm{~K}$ base temperature.

In Fig. 2, we present snapshots of the carrier distributions (upper panel) of bilayer graphene, along with the pumpinduced changes of the photocurrent (lower panel) for different pump-probe delays after excitation of the $E_{1 u}$ lattice vibration at $\lambda_{\text {pump }}=6.3 \mu \mathrm{m}$. These measurements were taken along the $\Gamma K$ direction, where only two of the four $\pi$ bands are visible due to photoemission matrix 
(a)

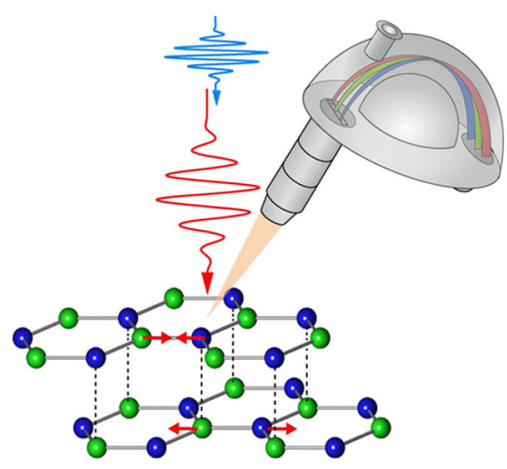

(b)

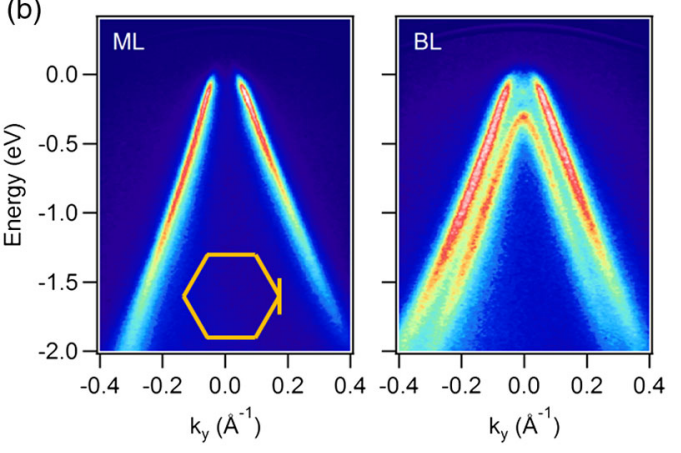

FIG. 1 (color online). (a) Sketch of the tr-ARPES experiment. The MIR-pump pulse (red, light grey) at normal incidence resonantly excites the in-plane $E_{1 u}$ lattice vibration in bilayer graphene. A collinear XUV-probe pulse (blue, dark grey) ejects photoelectrons that pass through a hemispherical analyzer and impinge on a two-dimensional detector. (b) Equilibrium band structure for hydrogen-intercalated monolayer (ML) and bilayer (BL) graphene measured with $\mathrm{HeIl} \alpha$ radiation for a cut through the $K$ point perpendicular to the $\Gamma K$ direction (see inset).

element effects [26]. Zero time delay was set to the peak of the pump-probe signal.

Consistent with previous measurements performed in the free carrier absorption regime [4], we observe a broadening of the Fermi edge due to an increase in electronic temperature, without signature of population inversion. The transient distributions of Fig. 2 were integrated over momentum, and plotted as a function of pump-probe delay [Fig. 3(a)]. At each time delay, the momentum-integrated photocurrent was fitted by a Fermi-Dirac distribution, to obtain the width of the Fermi cutoff, related to the electronic temperature, $T_{e}$. The time evolution of the Fermi width [Fig. 3(b)] is found to follow a double exponential decay, generally ascribed to the emission of optical $\left(\tau_{1}\right)$ and acoustic phonons $\left(\tau_{2}\right)$ [27-39].

Experiments for different excitation wavelengths were performed at a constant pump fluence of $F=0.26 \mathrm{~mJ} / \mathrm{cm}^{2}$ (see Supplemental Material [20]). The wavelength dependence of the peak electronic temperature, $\max T_{e}$, obtained by deconvolving the peak Fermi width with the experimental energy resolution (see Supplemental Material [20]), and the fast relaxation time, $\tau_{1}$, are plotted in Figs. 3(c) and 3(d), respectively. Both quantities exhibit a strong anomaly at the phonon resonance, with a reduced peak temperature and faster relaxation time $\left(\tau_{1}\right)$. The second relaxation time $\left(\tau_{2}=2.8 \pm 0.6 \mathrm{ps}\right)$ is instead found to be independent of pump wavelength.

Comparison with monolayer graphene (see Supplemental Material [20]), where the in-plane lattice vibration is not infrared active and hence cannot be excited with light [7], clearly indicates that the anomalies observed
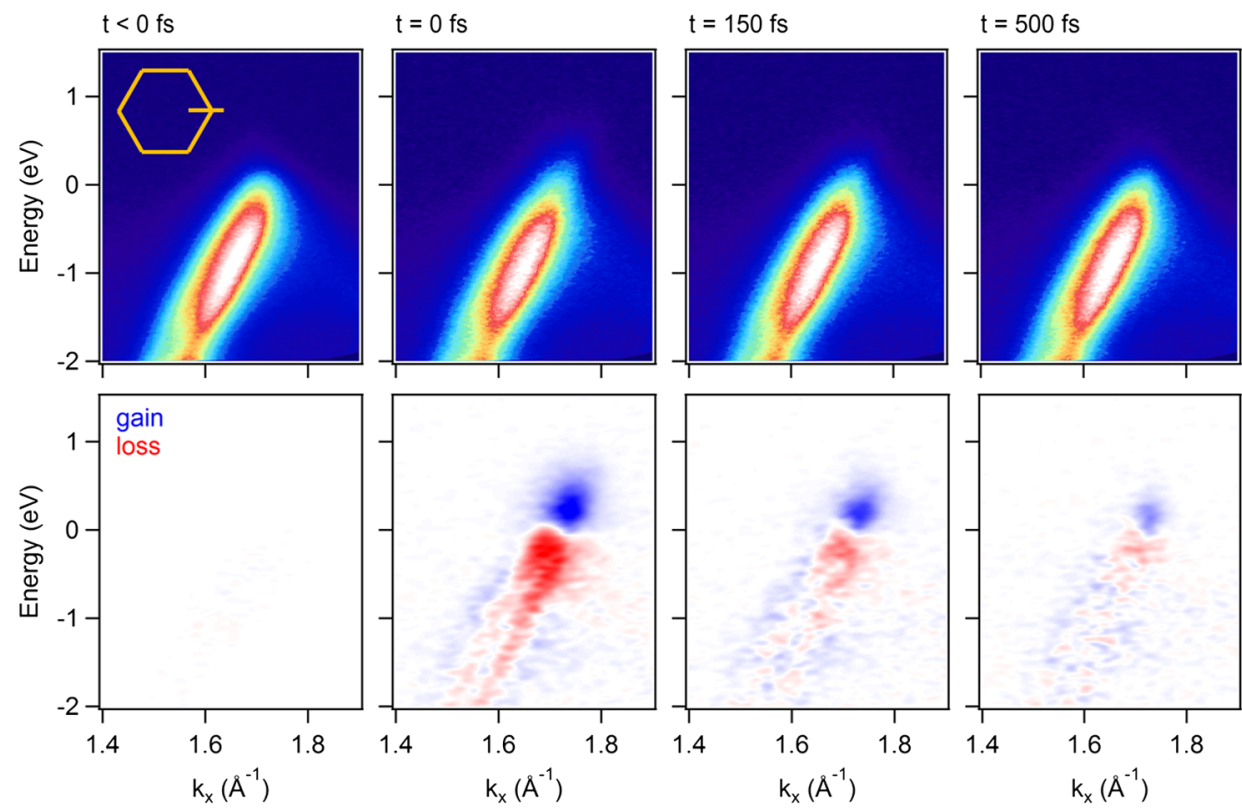

FIG. 2 (color online). Snapshots of the electronic structure along the $\Gamma K$ direction (see inset) of bilayer graphene for different pumpprobe delays (upper panel) together with the corresponding pump-probe signal (lower panel). The excitation wavelength was $6.3 \mu \mathrm{m}$ in resonance with the in-plane $E_{1 u}$ lattice vibration. The fluence was $F=0.26 \mathrm{~mJ} / \mathrm{cm}^{2}$. 

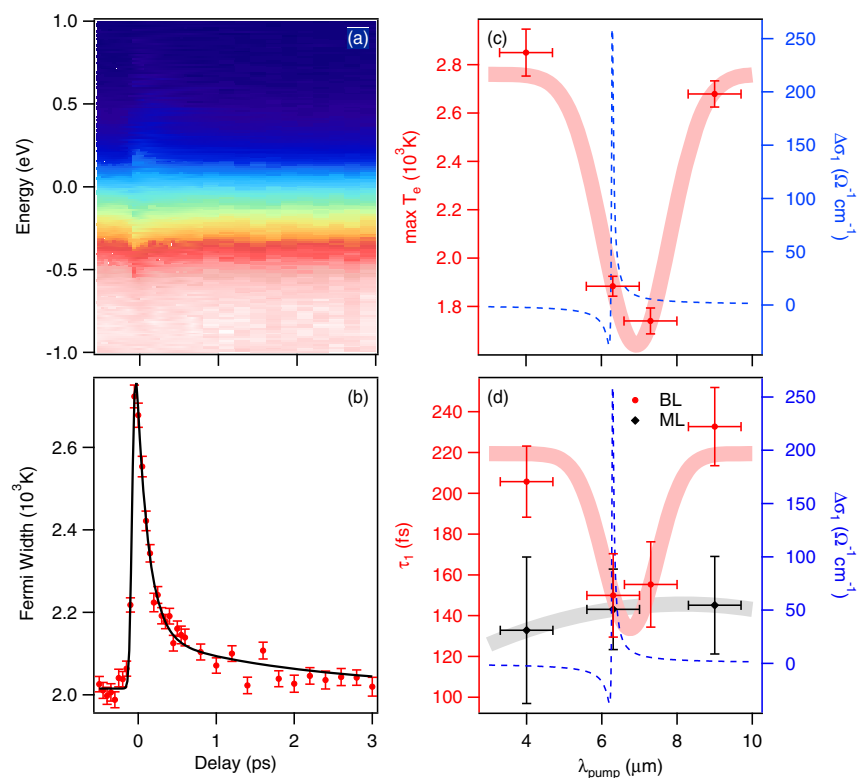

FIG. 3 (color online). (a) Momentum-integrated photocurrent from Fig. 2 as a function of energy and pump-probe delay in the vicinity of the Fermi level. (b) Width of the Fermi-Dirac distribution as a function of pump-probe delay (data points). The continuous line is a fit including an error function for the rising edge and the sum of two exponentials to describe the decay. (c) Dependence of the peak electronic temperature, obtained by deconvolving the peak Fermi width with the experimental energy resolution (see Supplemental Material [20]), on excitation wavelength for constant excitation fluence of $0.26 \mathrm{~mJ} / \mathrm{cm}^{2}$. (d) Dependence of the fast relaxation time $\tau_{1}$ on excitation wavelength for constant excitation fluence of $0.26 \mathrm{~mJ} / \mathrm{cm}^{2}$. The corresponding data for monolayer graphene for fluences between 0.26 and $0.8 \mathrm{~mJ} / \mathrm{cm}^{2}$ is shown in black for direct comparison. Continuous lines in (c) and (d) are guides to the eye. Dashed lines in (c) and (d) show the Fano line shape of the phonon in the real part of the optical conductivity $\Delta \sigma_{1}$ from [14].

in the bilayer system are not present. This is most obvious in Fig. 3(d), where we added the $\tau_{1}$ values for monolayer graphene (black data points) which are found to be independent of pump wavelength. We would like to point out that the SiC substrate exhibits two-phonon absorption in the wavelength range between 5.5 and $10 \mu \mathrm{m}$ [40]. Because of the weak van-der-Waals bond between ML or $\mathrm{BL}$ graphene and the $\mathrm{SiC}$ substrate, however, coherent phonon oscillations in the substrate resonantly excited by the pump pulse are unlikely to influence the carrier dynamics in the graphene layer.

The data presented above points then to important differences between excitation on and off resonance with the in-plane lattice vibration, which is interpreted in the following.

In the case of free carrier absorption [4], the excitation is a result of periodic acceleration and deceleration of the carriers along the direction of light polarization, $k_{y}[\Gamma M$ direction, green arrows in Fig. 4(a)]. Carrier heating occurs in this case because the Dirac carriers scatter during this oscillatory motion.

When excitation is resonant with the $E_{1 u}$ phonon, in addition to this time dependent polarization, one expects a modulation of the band structure, as the motion of the atoms makes the hopping between sites time dependent. Qualitatively, one can think of the motion of the atoms in real space directly translating into an oscillatory displacement of the Dirac cone itself [18]. Note that this motion of the Dirac cone occurs along $k_{x}[\Gamma K$ direction, blue arrows Fig. 4(a)], that is, perpendicular to the free carrier absorption occurring along $k_{y}$. Crucially, since the atomic motion is fast compared to the electron-phonon scattering time, the electrons do not follow the motion of the atoms promptly and experience a complex motion that breaks the adiabatic Born-Oppenheimer approximation [18].

To understand the physics at hand more quantitatively, we calculate the changes in the band structure for a static

(a)

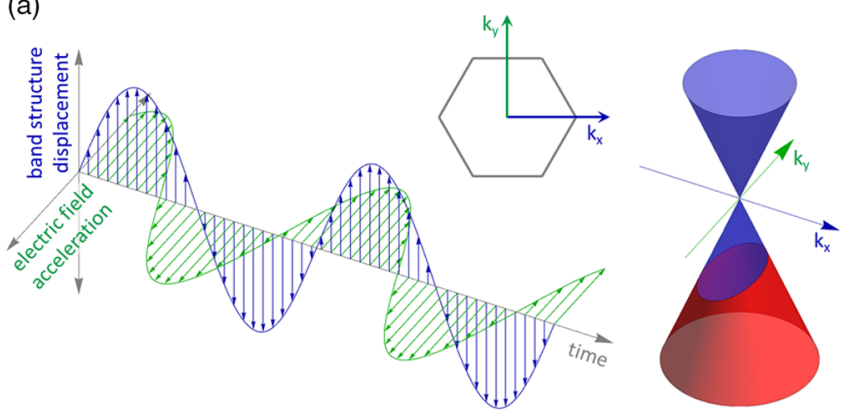

(b)

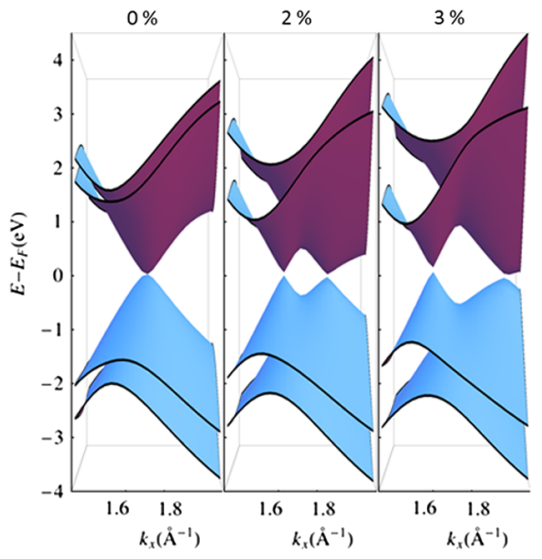

FIG. 4 (color online). (a) Sketch of the excitation due to electric field acceleration (green, light grey) and band structure shift (blue, dark grey). The field polarization lies along $k_{y}(\Gamma M)$. The displacement of the atoms in real space leads to a corresponding displacement of the Dirac cone in momentum space along $k_{x}(\Gamma K)$. The combined effect results in a complicated sloshing motion of the electrons that depends on the relative phase and amplitudes of the electric field acceleration and the band structure displacement, respectively. (b) Density functional theory calculations of the electronic structure around the $K$ point in the vicinity of the Fermi level for different lattice distortions of $0 \%, 2 \%$, and $3 \%$ of the equilibrium lattice constant along the $E_{1 u}$ mode coordinate. 
lattice distortion along the $E_{1 u}$ normal mode coordinate. For a pump field of approximately $1 \mathrm{MV} / \mathrm{cm}$, as employed in our experiments, we estimate atomic displacements of $\sim 5 \mathrm{pm}, \sim 2 \%$ of the equilibrium lattice constant (see Supplemental Material [20]). In Fig. 4(b), we report calculated band structures for different lattice distortions along the $E_{1 u}$ normal mode coordinate. Dramatic changes are induced by the vibration, with a momentum splitting of the $\pi$ bands at the $K$ point and a huge shift of the $\sigma$ bands at the $\Gamma$ point towards the Fermi level (see Supplemental Material [20]). The oscillatory motion of the Dirac cone known from the monolayer [Fig. 4(a)] affects the upper and lower layer of the bilayer in opposite directions. Together with the interlayer coupling this leads to a splitting of the $\pi$ bands along $\Gamma \mathrm{KM}$ that increases with increasing distortion [Fig. 4(b)]. The nonequilibrium occupancy of the transient electronic states is determined by the complex nonadiabatic motion sketched in Fig. 4(a).

To describe the experimental results we consider how the dynamical changes in the density of states (see Fig. 4 in the Supplemental Material [20]) are expected to affect the electronic temperature. In order to approximately estimate the change in peak electronic temperature due to the $E_{1 u}$ lattice distortion, we assume that during the lattice distortion both the electron number $N$ (DOS, $\mu_{e}, T_{e}$ ) and the entropy of the system $S$ (DOS, $\mu_{e}, T_{e}$ ) are conserved (see Supplemental Material [20]). The chemical potential and the peak electronic temperature in the absence of a lattice distortion are known from off-resonance tr-ARPES data $\left(\mu_{0} \sim-200 \mathrm{meV}, T_{0} \sim 2800 \mathrm{~K}\right.$ ). Based on the DOS (see Fig. 4 in the Supplemental Material [20]), we obtain $\mu_{1} \sim$ $-350 \mathrm{meV}$ and $T_{1} \sim 1600 \mathrm{~K}$ for an atomic displacement of $3 \%$ of the lattice constant, an estimate that can be reconciled with the data. One may speculate further that the changes in electronic DOS affect the electron-phonon coupling constant and result in faster carrier relaxation.

In summary, we have excited monolayer and bilayer graphene at MIR wavelengths between 4 and $9 \mu \mathrm{m}$, both on and off resonance with the in-plane $E_{1 u}$ mode in bilayer graphene, and probed the response of the electronic structure with tr-ARPES. We find that both the peak electronic temperature as well as the relaxation rate are significantly perturbed when the excitation is made resonant with the $E_{1 u}$ mode, an effect that is absent in monolayer graphene, in which light cannot couple to the in-plane lattice vibration. We explain the data by discussing the dynamical band structure changes combined with a nonadiabatic temporal response, a genuinely new type of carrier excitation for the solid state. Similar concepts may be extended beyond graphene, for example, to the transition metal dichalcogenides, opening up new avenues for electronic structure control with light. The complex circular motion of the electrons throughout momentum space, which involves ultrafast manipulation of the electronic structure of the system over very few femtoseconds, may be interesting for applications in optoelectronics at very high bit rates.

We thank Jörg Harms for assisting with the figures and Axel Köhler for hydrogen etching and argon annealing of the samples. Access to the Artemis facility at the Rutherford Appleton Laboratory was funded by STFC. A.L. acknowledges financial support from the German Science Foundation (DFG, SFB 925) and the EU-Flagship Graphene.

*Isabella.Gierz@mpsd.mpg.de

[1] J. C. Johannsen, S. Ulstrup, F. Cilento, A. Crepaldi, M. Zacchigna, C. Cacho, I. C. Edmond Turcu, E. Springate, F. Fromm, C. Raidel, T. Seyller, F. Parmigiani, M. Grioni, and P. Hofmann, Phys. Rev. Lett. 111, 027403 (2013).

[2] S. Ulstrup, J. C. Johannsen, F. Cilento, J. A. Miwa, A. Crepaldi, M. Zacchigna, C. Cacho, R. Chapman, E. Springate, S. Mammadov, F. Fromm, C. Raidel, T. Seyller, F. Parmigiani, M. Grioni, P. D. C. King, and P. Hofmann, Phys. Rev. Lett. 112, 257401 (2014).

[3] T. Li, L. Luo, M. Hupalo, J. Zhang, M. C. Tringides, J. Schmalian, and J. Wang, Phys. Rev. Lett. 108, 167401 (2012)

[4] I. Gierz, J. C. Petersen, M. Mitrano, C. Cacho, I. C.E. Turcu, E. Springate, A. Stöhr, A. Köhler, U. Starke, and A. Cavalleri, Nat. Mater. 12, 1119 (2013).

[5] T. Winzer, E. Malić, and A. Knorr, Phys. Rev. B 87, 165413 (2013).

[6] S. Winnerl, M. Orlita, P. Plochocka, P. Kossacki, M. Potemski, T. Winzer, E. Malic, A. Knorr, M. Sprinkle, C. Berger, W. A. de Heer, H. Schneider, and M. Helm, Phys. Rev. Lett. 107, 237401 (2011).

[7] A. C. Ferrari and D. M. Basko, Nat. Nanotechnol. 8, 235 (2013).

[8] T. Ando, J. Phys. Soc. Jpn. 76, 104711 (2007).

[9] L. M. Malard, D. C. Elias, E. S. Alves, and M. A. Pimenta, Phys. Rev. Lett. 101, 257401 (2008).

[10] T. Ando and M. Koshino, J. Phys. Soc. Jpn. 78, 034709 (2009).

[11] P. Gava, M. Lazzeri, A. M. Saitta, and F. Mauri, Phys. Rev. B 80, 155422 (2009).

[12] N. Bonini, M. Lazzeri, N. Marzari, and F. Mauri, Phys. Rev. Lett. 99, 176802 (2007).

[13] C.-H. Park, F. Giustino, M. L. Cohen, and S. G. Louie, Nano Lett. 8, 4229 (2008).

[14] A. B. Kuzmenko, L. Benfatto, E. Cappelluti, I. Crassee, D. van der Marel, P. Blake, K. S. Novoselov, and A. K. Geim, Phys. Rev. Lett. 103, 116804 (2009).

[15] Y. Zhang, T.-T. Tang, C. Girit, Z. Hao, M. C. Martin, A. Zett, M. F. Crommie, Y. R. Shen, and F. Wang, Nature (London) 459, 820 (2009).

[16] T.-T. Tang, Y. Zhang, C.-H. Park, B. Geng, C. Girit, Z. Hao, M. C. Martin, A. Zettl, M. F. Crommie, S. G. Louie, Y. R. Shen, and F. Wang, Nat. Nanotechnol. 5, 32 (2010).

[17] E. Cappelluti and G. Profeta, Phys. Rev. B 85, 205436 (2012). 
[18] S. Pisana, M. Lazzeri, C. Casiraghi, K. S. Novoselov, A. K. Geim, A. C. Ferrari, and F. Mauri, Nat. Mater. 6, 198 (2007).

[19] C. Riedl, C. Coletti, T. Iwasaki, A. A. Zakharov, and U. Starke, Phys. Rev. Lett. 103, 246804 (2009).

[20] See Supplemental Material at http://link.aps.org/ supplemental/10.1103/PhysRevLett.114.125503, which includes Refs. [21-25], for experimental and theoretical methods, sample preparation, additional experimental data for monolayer and bilayer graphene at different pump wavelengths, and frozen phonon calculations for complete band structure and density of states.

[21] G. Kresse and J. Hafner, J. Phys. Condens. Matter 6, 8245 (1994).

[22] P. E. Blöchl, Phys. Rev. B 50, 17953 (1994).

[23] G. Kresse and D. Joubert, Phys. Rev. B 59, 1758 (1999).

[24] P. E. Blöchl, O. Jepsen, and O. K. Andersen, Phys. Rev. B 49, 16223 (1994).

[25] A. F. Kemper, M. A. Sentef, B. Moritz, J. K. Freericks, and T. P. Devereaux, Phys. Rev. B 90, 075126 (2014).

[26] E. L. Shirley, L. J. Terminello, A. Santoni, and F. J. Himpsel, Phys. Rev. B 51, 13614 (1995).

[27] M. Breusing, S. Kuehn, T. Winzer, E. Malić, F. Milde, N. Severin, J. P. Rabe, C. Ropers, A. Knorr, and T. Elsaesser, Phys. Rev. B 83, 153410 (2011).

[28] C. H. Lui, K. F. Mak, J. Shan, and T. F. Heinz, Phys. Rev. Lett. 105, 127404 (2010).
[29] T. Kampfrath, L. Perfetti, F. Schapper, C. Frischkorn, and M. Wolf, Phys. Rev. Lett. 95, 187403 (2005).

[30] K. Kang, D. Abdula, D. G. Cahill, and M. Shim, Phys. Rev. B 81, 165405 (2010).

[31] E. Malic, T. Winzer, E. Bobkin, and A. Knorr, Phys. Rev. B 84, 205406 (2011).

[32] G. Moos, C. Gahl, R. Fasel, M. Wolf, and T. Hertel, Phys. Rev. Lett. 87, 267402 (2001).

[33] J. M. Dawlaty, S. Shivaraman, M. Chandrashekhar, F. Rana, and M. G. Spencer, Appl. Phys. Lett. 92, 042116 (2008).

[34] B. Gao, G. Hartland, T. Fang, M. Kelly, D. Jena, H Xing, and L. Huang, Nano Lett. 11, 3184 (2011).

[35] D. Sun, C. Divin, C. Berger, W. A. de Heer, P. N. First, and T. B. Norris, Phys. Rev. Lett. 104, 136802 (2010).

[36] M. Breusing, C. Ropers, and T. Elsaesser, Phys. Rev. Lett. 102, 086809 (2009).

[37] P. A. George, J. Strait, J. Dawlaty, S. Shivaraman, M. Chandrashekhar, F. Rana, and M. G. Spencer, Nano Lett. 8, 4248 (2008).

[38] L. Huang, G. V. Hartland, L.-Q. Chu, Luxmi, R. M. Feenstra, C. Lian, K. Tahy, and H. Xing, Nano Lett. 10, 1308 (2010).

[39] P. J. Hale, S. M. Hornett, J. Moger, D. W. Horsell, and E. Hendry, Phys. Rev. B 83, 121404(R) (2011).

[40] S. A. Alekseev, V. N. Zaitsev, J. Botsoa, and D. Barbier, Chem. Mater. 19, 2189 (2007). 